\title{
Heavy quarks at HERA with lifetime tag
}

\author{
Massimo Corradi* ${ }^{\dagger}$ \\ INFN Bologna, Italy \\ E-mail: corradi@mail.desy.de
}

Two preliminary measurements of beauty production in events with two jets at HERA are presented. The first exploits the new ZEUS microvertex detector to measure the $b$ content of a sample of events with two jets and a muon. The second is a measurement by the H1 collaboration based on inclusive lifetime tagging. The results are in agreement with previous measurements and with QCD predictions.

International Europhysics Conference on High Energy Physics

July 21st - 27th 2005

Lisboa, Portugal

${ }^{*}$ Speaker.

${ }^{\dagger}$ on behalf of the H1 and ZEUS collabotrations 


\section{Introduction}

The production of beauty quarks in $e p$ collisions is a stringent test of perturbative QCD since the large quark mass provides a hard scale that should make perturbative calculations reliable. The ZEUS and $\mathrm{H} 1$ collaborations already published $[1,3,2]$ the measurements of beauty production in events with two jets and a muon in $e p$ collision at $\sqrt{s}=320 \mathrm{GeV}$ at HERA. The production cross section was found to be consistent with next-to-leading order (NLO) QCD calculations, with the exception of a possible excess over theory found by $\mathrm{H} 1$ at the lowest measured transverse momentum of the muon $\left(2.5<p_{T}^{\mu}<3 \mathrm{GeV}\right)$. The determination of the beauty content of the dijet sample is the limiting factor in the precision of this kind of measurements. The beauty content was measured by the ZEUS collaboration using the transverse momentum of the muon with respect to the nearest jet, $p_{T}^{\text {rel }}$, which is expected to be, on average, larger for beauty than for charm and light flavours (LF) due to the large $b$ mass. The H1 collaboration, thanks to its silicon vertex detector, could also exploit a precise measurement of the distance of closest approach of the muon track from the primary vertex to distinguish muons coming from the decay of long-lived heavy-flavoured hadrons from backgrounds, in combination with the measurement of $p_{T}^{\text {rel }}$. The ZEUS collaboration installed a microvertex detector (MVD) during the HERA luminosity upgrade (HERA-II) and can therefore exploit lifetime tagging in HERA-II data. Two preliminary results on beauty production in dijet events are presented here: the first ZEUS measurement that uses the new silicon vertex detector [4] and a measurement by $\mathrm{H} 1$ based on an inclusive lifetime tagging technique that does not require leptons [5].

\section{ZEUS results with HERA-II data}

Since fall 2003 ZEUS has been collecting data with the new silicon microvertex detector. The analysis presented here is based on an integrated luminosity of $33 \mathrm{pb}^{-1}$ of $e^{+} p$ collisions taken in the year 2004. Photoproduction (i.e. $Q^{2}<1 \mathrm{GeV}^{2}$ ) events with two jets with $p_{T}^{j_{1}\left(j_{2}\right)}>7(6) \mathrm{GeV}$ and a muon with $p_{T}^{\mu}>2.5 \mathrm{GeV}$ associated to one of the two jets were selected, giving a sample
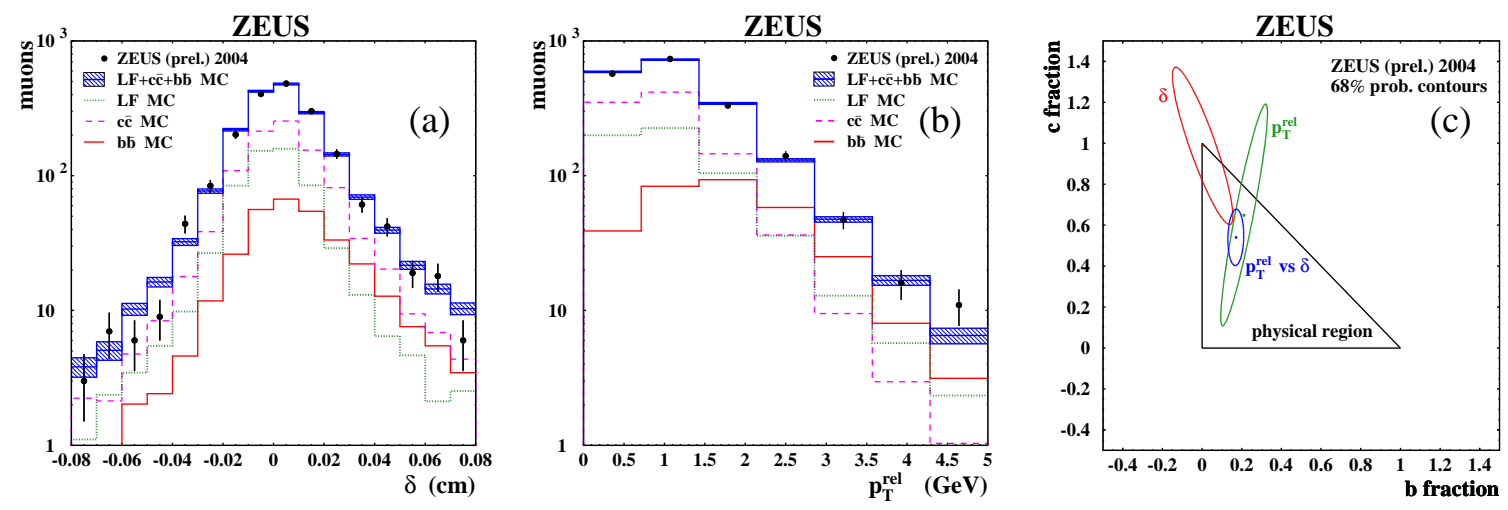

Figure 1: ZEUS analysis of HERA-II data. The distributions of the muon impact parameter $\delta$ (a) and $p_{T}^{\text {rel }}$ (b) are compared to the sum of MC templates for beauty $(b \bar{b})$, charm $\left(c^{-} c\right)$ and light fhvour (LF) events. The small ellipse in (c) represents the $68 \%$ probability contour in the plane defined by the charm and beauty fraction as obtained from a fit to $\delta$ and $p_{T}^{\text {rel }}$. The large ellipses are the result of single-variable fits. 


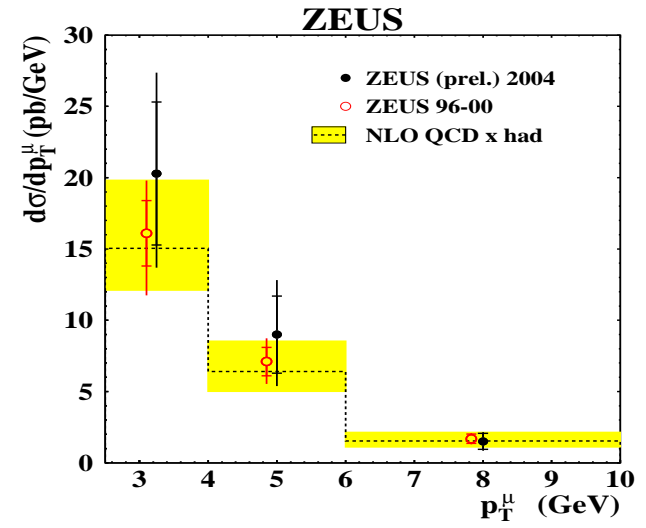

Figure 2: Differential cross-section as a function of the muon transverse momentum $p_{T}^{\mu}$ for beauty production in two-jet events with $Q^{2}<1 \mathrm{GeV}^{2}$, $0.2<y<0.8, p_{T}^{j_{1}\left(j_{2}\right)}>7(6) \mathrm{GeV}, \eta^{j_{1}\left(j_{2}\right)}<2.5$ and $-1.6<\eta^{\mu}<2.3$. The ZEUS HERA-II data are compared to the HERA-I measurement and to the nextto-leading order QCD prediction with hadronization corrections.

of 1806 events. The flavour content was analysed by means of the muon $p_{T}^{\text {rel }}$ and $\delta$, the distance of closest approach of the muon track to the average beam position in the transverse plane. A positive sign was assigned to $\delta$ if the muon track crossed the jet axis downstream of the beam position in the transverse plane, negative otherwise. The distribution of the two variables is shown if Fig. 1 $(a, b)$ and compared to Monte Carlo templates for beauty, charm and light flavours. The excess at positive $\delta$ shows the presence of muons coming from a displaced vertex from $c$ and $b$ decays. The beauty content was obtained by fitting the two-dimensional distribution of the data in $p_{T}^{\text {rel }}$ and $\delta$ with the MC templates. Figure 1(c) shows the $68 \%$ probability contours in the plane defined by the $c$ and $b$ fractions as obtained from the fit to the two-dimensional distribution and from separate fits to each variable. The $b$ fraction from the two-dimensional fit, which gives the best precision, is $f_{b}=(16.7 \pm 2.6) \%$. Though the understanding and the alignment of the MVD are still preliminary, the distance of closest approach improves apparently the determination of the charm and beauty content. Figure 2 shows the measured cross section for $e p \rightarrow b \bar{b} X \rightarrow j j \mu X^{\prime}$ as a function of the muon transverse momentum $p_{T}^{\mu}$. The result from HERA-II data is compared to the HERA-I measurement [3], based on a $\sim 3$ times larger integrated luminosity, and to the NLO QCD prediction. The agreement is good and there is no hint for an excess at low $p_{T}^{\mu}$ such as that suggested by $\mathrm{H} 1$ [2].

\section{H1 results with inclusive lifetime tagging}

The H1 collaboration has developed [6] a technique for the flavour analysis of jets based on the impact parameter significance $S$ of the tracks associated to the jet, where $S$ is defined as the distance of closest approach of a track to the event vertex divided by its uncertainty: $S=\delta / \sigma_{\delta}$. This technique has been applied to a sample of dijet events with $p_{T}^{j_{1}\left(j_{2}\right)}>11(8) \mathrm{GeV}$ and $Q^{2}<1 \mathrm{GeV}^{2}$ collected during the '99-2000 running period and corresponding to an integrated luminosity of $57.7 \mathrm{pb}^{-1}$. Since the probability for finding two tracks with large impact parameter significance in the LF background is small, the second-highest significance $S_{2}$ was used to separate the $b, c$ and LF components. Figure 3 shows the distribution of $S_{2}$ with the positive tail due to long-lived $c$ and $b$ hadrons contained in the jet. This method is sufficiently powerful to allow the extraction of the beauty and charm fraction in a sample that was not enriched in heavy flavours by requiring muons. The measured charm and beauty cross sections for $e p \rightarrow e^{\prime} Q \bar{Q} X \rightarrow e^{\prime} j_{1} j_{2} X^{\prime}$ are shown in Fig.4 


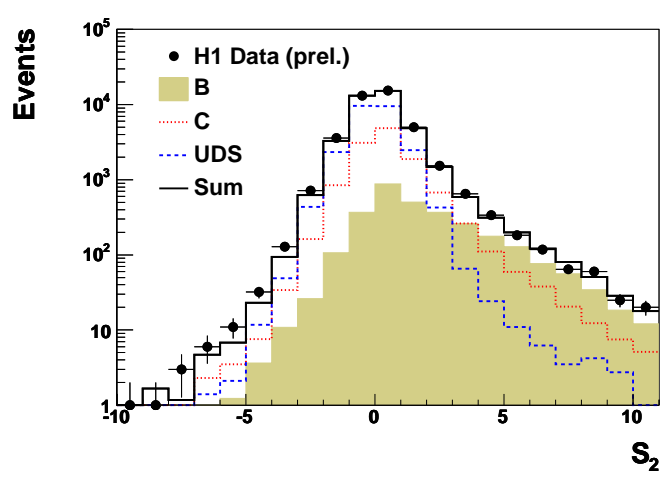

Figure 3: Distribution of the impact parameter significance $S$ for the second-highest $S$ track in the H1 dijet sample. The data (points) are compared to the sum of MC templates for beauty (B), charm (C) and light-fhvour (UDS) events.
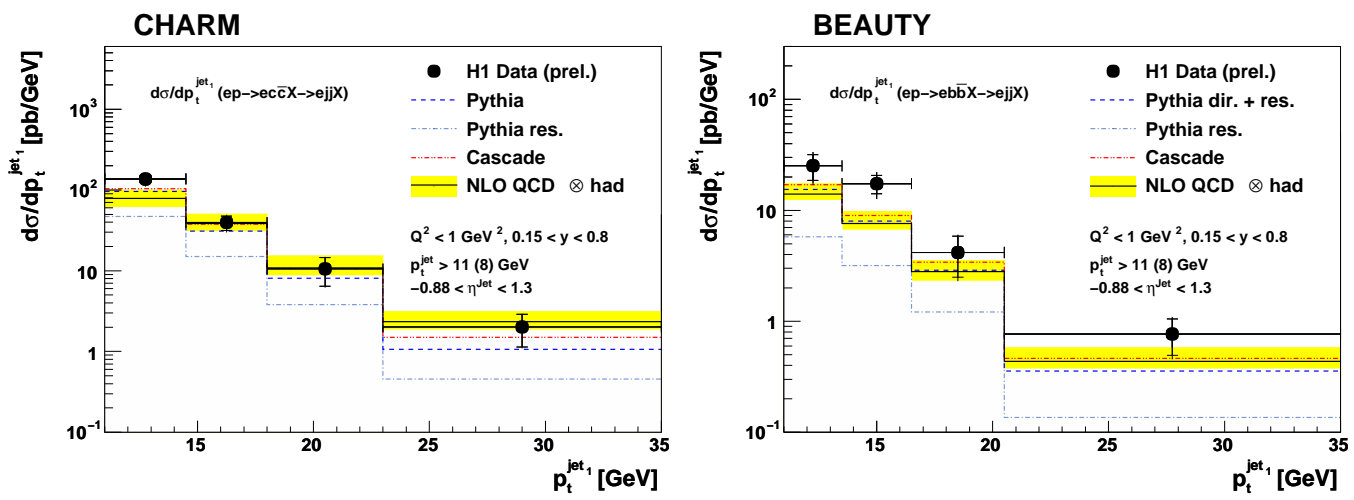

Figure 4: Differential cross-section as a function of transverse momentum of the highest $p_{T}$ jet for charm (left) and beauty (right) dijet events with $Q^{2}<1 \mathrm{GeV}^{2}, 0.2<y<0.8, p_{T}^{j_{1}\left(j_{2}\right)}>11(8) \mathrm{GeV}$ and $-0.88<\eta^{j_{1}\left(j_{2}\right)}<1.3$. The H1 data (points) are compared to the next-to-leading order QCD predictions with hadronization correction (band) and to the MC models Pythia and Cascade.

as a function of the transverse momentum of the highest- $p_{T}$ jet and compared to the NLO QCD prediction. Charm is in good agreement with the theoretical prediction. The beauty cross-section is somewhat larger than the NLO QCD prediction in the first two bins $\left(11<p_{T}^{j_{1}}<15.5 \mathrm{GeV}\right)$, though still compatible within two standard deviations. Considering that the other analyses found good agreement in this range, this excess can be probably ascribed to a fluctuation.

\section{References}

[1] B. Naroska, these proceedings;

[2] A. Aktas et al. (H1 coll.), Eur. Phys. J. C41 (2005) 453-467;

[3] S. Chekanov et al. (ZEUS coll.), Phys. Rev. D 70 (2004) 012008;

[4] ZEUS collaboration, contributed paper to EPS-HEP2005, Abstract 359;

[5] H1 collaboration, contributed paper to the XXII International Symposium on Lepton-Photon Interactions at High Energy (Uppsala 2004), Abstract 405;

[6] A. Aktas et al. (H1 coll.), Eur. Phys. J. C40 (2005) 349-359. 